\title{
De-adhesion dynamics of melanoma cells from brain endothelial layer
}

\author{
Béla Varga ${ }^{1,3}$, Réka Anita Domokos ${ }^{1,2}$ Csilla Fazakas $^{1}$, Imola Wilhelm ${ }^{1,4}$, István A. \\ Krizbai $^{1,4}$, Zsolt Szegletes $^{1}$, Csilla Gergely ${ }^{3}$, György Váró $^{1}$ and $\underline{\text { Attila G. Végh }}{ }^{\mathbf{1}}$
}

${ }^{1}$ Institute of Biophysics, Biological Research Centre, Hungarian Academy of Sciences, Szeged, Hungary

${ }^{2}$ Babes-Bolyai University, Faculty of Physics, Cluj-Napoca, Romania

${ }^{3}$ Laboratoire Charles Coulomb, UMR 5221 CNRS - Université de Montpellier, Montpellier, France

${ }^{4}$ Institute of Life Sciences, Vasile Goldiş Western University, Arad, Romania

Corresponding author: Attila G. Végh PhD

BRC Temesvári krt 62, Szeged, Hungary, H-6726

E-mail: vegh.attilagergely@brc.mta.hu 


\begin{abstract}
Metastasis formation is a complex and not entirely understood process. The poorest prognosis and the most feared complications are associated to brain metastases. Melanoma derived brain metastases show the highest prevalence. Due to the lack of classical lymphatic drainage, in the process of brain metastases formation the haematogenous route is of primordial importance. The first and crucial step in this multistep process is the establishment of firm adhesion between the blood travelling melanoma cells and the tightly connected layer of the endothelium, which is the fundamental structure of the blood-brain barrier. This study compares the de-adhesion properties and dynamics of three melanoma cells types (WM35, A2058 and A375) to a confluent layer of brain micro-capillary endothelial cells. Cell type dependent adhesion characteristics are presented, pointing towards the existence of metastatic potential related nanomechanical aspects. Apparent mechanical properties such as elasticity, maximal adhesion force, number, size and distance of individual rupture events showed altered values pointing towards cell type dependent aspects. Our results underline the importance of mechanical details in case of intercellular interactions. Nevertheless, it suggests that in adequate circumstances elastic and adhesive characterizations might be used as biomarkers.
\end{abstract}

Keywords: atomic force microscope, single-cell force spectroscopy, cell mechanics, melanoma cell adhesion, cerebral endothelial cell, metastatic potential

\title{
Introduction
}

Occurrence of melanoma has increased worldwide, being responsible for over $80 \%$ of skin cancer deaths (1). Although metastatic melanoma has relatively low abundance, it shows high resistance to conventional therapies $(2,3)$. Metastasis to brain is difficult to treat; local surgery, whole brain radio therapy, stereotactic radiosurgery have been the only treatment approaches for a long time (4). Recently, incorporation of systemic treatments such as molecularly targeted therapies and immunotherapies have emerged as alternatives (5). Although results of these therapies seem to be promising, at the opposite side, prevention might be important as well. In order to find effective prevention strategies, lacking details of brain metastasis formation needs to be elucidated.

The first obstacle for hematogenously disseminated melanoma cells to enter the brain parenchyma is the tight layer of brain endothelial cells, forming the blood-brain barrier (BBB)(6). The first step in the highly orchestrated process of melanoma cell transmigration over the brain endothelium is the establishment of firm connection. Several environmental and molecular factors play crucial role in this process which manifests in the arrest of melanoma cells at the luminal surface of the blood vessels (7). Dynamics of this first but crucial adhesive step might reveal important information about its nature and characteristics.

Atomic force microscopy (AFM) based single-cell force spectroscopy is an emerging method to directly investigate cellular mechanics and the dynamics of intercellular interactions $(8,9)$. Excluding the immobilization of the melanoma cell, during these measurements there is no need for any staining or other cell-life impairing preparation. Short term (up to a few tens of seconds) 
adhesive properties can be studied upon analysis of the detaching process of individual cells. Due to the high force-resolution provided by the AFM besides maximal adhesion forces, individual deadhesion events can be identified and compared accurately, which are the hallmark of the established connection $(10,11)$. Furthermore, even topographical mapping can be reconstructed based on adhesive data, which might reveal alterations at sub cellular level (12).

Basically every active and passive connection might contribute to the established adhesive strength between a blood travelling tumour cell and the endothelial cells. It has been reported, that in case of breast cancer cells the elasticity is inversely proportional to the inter-homocellular adhesion which decreases with metastatic potential (13). Expression of cell adhesion molecules in endothelial cells influences their adhesive properties to bladder cancer cells (14). Cell-cell interaction strength might be modulated by extracellular protonation too, hence it plays an important role in tissue invasion of melanoma cells (15). The thickness of the outer glycocalyx layer on the surface of the endothelial cells is influencing their adhesive properties. Short term hyperglycaemia induces thickening of glycocalyx layer and higher adhesion between lung carcinoma and human aorta endothelial cells (16).

Cell adhesion depends on multiple and even multivariate individual molecular connections, where the individual players are difficult to identify. On single-cell force spectroscopy based mechanical assumptions, hereby we present our latest data on the interaction of three different types of melanoma cells, having altered invasive characteristics, with brain endothelial cells. Our results show that nanomechanical properties can be associated to higher metastatic potential and invasive characteristics may rely on stronger adhesive properties mediated by altered tether formation dynamics.

\section{Materials and Methods}

\section{Cell culture}

hCMEC/D3 human microvascular cerebral endothelial cells (shortly D3 (17)) were grown on rat tail collagen-coated dishes in EBM-2 medium (Lonza) supplemented with EGM-2 Bullet Kit (Lonza).

Highly invasive A2058 human melanoma cells (obtained from the European Collection of Cell Cultures) were maintained in EMEM (Sigma) supplemented with 5\% FBS (Sigma). The A2058 cells were derived from metastatic site (lymph node) of a 43 years old Caucasian man. A375 human metastatic melanoma cells were derived from skin and the less invasive WM35 human primary melanoma cells were cultured in DMEM (GIBCO), supplemented with $5 \%$ FBS. During culturing, cells were kept at 5\% $\mathrm{CO}_{2}$ atmosphere at $37^{\circ} \mathrm{C}$.

All three cell types are BRAF: V600E mutant, NRAS wild type. Melanoma cells were labelled with CellTracker ${ }^{\mathrm{TM}}$ Red CMTPX Dye (Life Technologies), ensuring that they can be clearly identified prior to immobilization at the end of the cantilever.

\section{Atomic Force Microscope}

All experiments were performed with an Asylum Research MFP-3D atomic force microscope (Asylum Research, Santa Barbara, CA; driving software written in IgorPro 6.34A, Wavemetrics), mounted on the top of a Zeiss 
Axiovert 200 optical microscope for optical positioning.

The experiments were carried out with rectangular tipless gold coated cantilevers, having a nominal spring constant of $50 \mathrm{pN} / \mathrm{nm}$, resonant frequency of $10 \mathrm{kHz}$ in air (MikroMasch, Tallinn, Estonia), which drops to $3 \mathrm{kHz}$ in water. The spring constant of the cantilever was determined each time by thermal calibration (18-20).

Cancer cells were immobilized at the very end of the tipless cantilever using a Concanavalin-A mediated linkage which is described in details elsewhere $(21,22)$. All experiments were conducted in serum free Leibovitz medium at $37^{\circ} \mathrm{C}$ within two hours after the cells were taken out from the incubator. According to our experience, they preserve their viability far beyond this period.

\section{Single Cell Force Spectroscopy}

During a force measurement cycle, a cancer cell immobilized at the end of a tipless cantilever was brought into contact with surface-adherent endothelial cells. The schematic representation can be found in Figure 1.

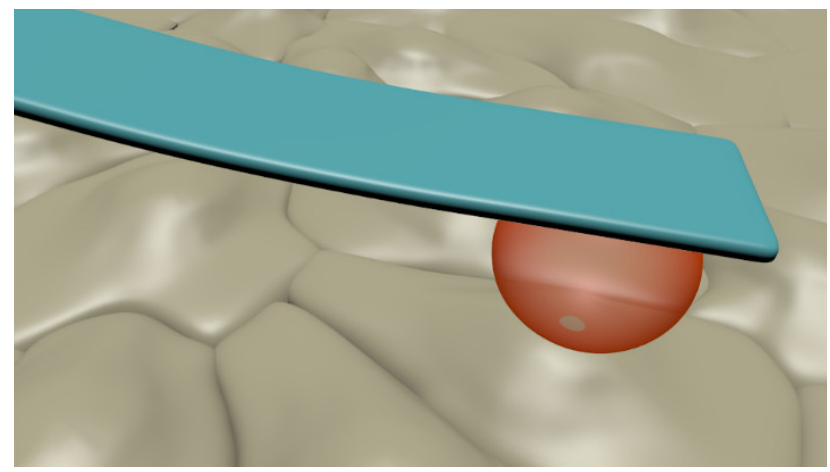

Figure 1. Schematic representation of experimental arrangement. Melanoma cell labelled with CellTracker $^{\mathrm{TM}}$ Red dye immobilized at the end of a tipless cantilever, pushed to a confluent layer of endothelial cells.

Each cycle consisted of approaching the cellloaded cantilever to the endothelial layer until the pre-set deflection was reached and pulling it back to initial position. Force curves were recorded at constant loading speed $(2 \mu \mathrm{m} / \mathrm{s})$ and sampling frequency $(2 \mathrm{kHz})$. Total force distance was kept at $8 \mu \mathrm{m}$ with maximum load of $2.0 \mathrm{nN}$. After the maximal load was reached, a dwell time of 3 seconds was applied before the retraction of the cantilever.

Figure 2 shows a typical force-distance curve: point of contact is marked with "o", approaching phase is drawn in red while blue represents retraction, as pointed by accordingly coloured arrows.

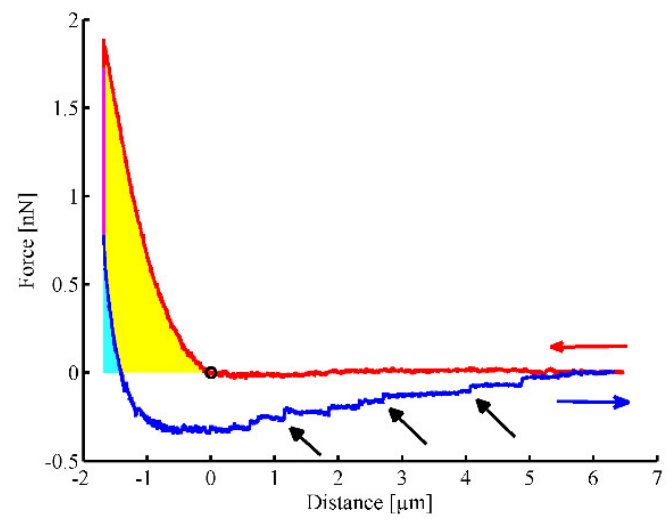

Figure 2. A representative force-distance curve. Trace is drawn in red, retrace in blue. Contact point is marked with "o", cyan area marks the remanent work, cyan + yellow area marks the total work, black arrows indicate the place of located rupture events.

\section{Data analysis}

A homemade MATLAB routine was used to extract all parameters. A relative elasticity, similar to the elasticity index in case of polymers (23), was calculated as the ratio between the remanent work (cyan area, Figure 2) and the total work (cyan + yellow area, Figure 2). This parameter can take values between 0 and 1 , where 0 represents perfectly plastic while 1 perfectly elastic behaviour.

Values of maximal adhesion force were considered as the difference between the force at the maximal downward deflections of the cantilever compared to the initial value during non-contact state. Disruption events were identified as sudden deflection changes during retraction. Only those were considered where the level difference was higher than threefold the standard deviation at the end of the respective curve. Rupture size was calculated as a level difference of mean of 5 point before and after the place of occurrence. Rupture length represents the distance from the place of maximal adhesion 
force (maximal deflection) and the position of the last point before an identified disruption event.

Distribution of rupture size and length are represented by histograms showing the probability to find a given value within the whole data population calculated for each cell type. Using this representation, the eventual distortions due to different abundances can be avoided. In case of rupture length, a logarithmic scale was chosen to balance the unequal representation for abundant and rare events.

The calculated values for relative elasticity, maximal adhesion force and number of rupture events are presented with the help of "box plots". In order to cover the level, spread and symmetry of the data values, box plots show the median, the approximate quartiles and the lowest and highest data points (24). This representation has the advantage to show eventual skewness in the distribution of the values, while treats extreme values as outliers: in our case 1.5 fold the interquartile range away from the top or bottom of the box was considered as outlier and marked with "plus" signs.

Experiments were repeated more than five times for each type of melanoma cell, in each case 40 curves were recorded with the very same pre-set parameters.

\section{Results}

Adhesion of three different types of human melanoma cells to a confluent layer of human brain endothelial cells was measured. In our dynamic experiments the three types of melanoma cells of different metastatic characteristics were represented by WM35, A2058 and A375 cell lines, while the endothelial layer was formed by the hCMEC/D3 (shortly D3) cell line. As an internal comparison in each case prior to measuring intercellular interactions the very same melanoma cells were contacted to a cell free area of the Petri dish as well. Same parameters (load, force speed, dwell time in contact) were used in case of all cell types.

In order to achieve a more illustrative comparison of elasticity, a relative dimensionless parameter was used (see section of Materials and Methods for details). Figure 3 shows the calculated relative elasticity of the studied cell types. Similar dependence pattern can be observed when the melanoma cells are pushed to bare Petri dish and to endothelial cells. This underlines that the measured parameters reflect mainly the characteristics of the melanoma cells, hence the endothelial layers can be considered practically invariable.

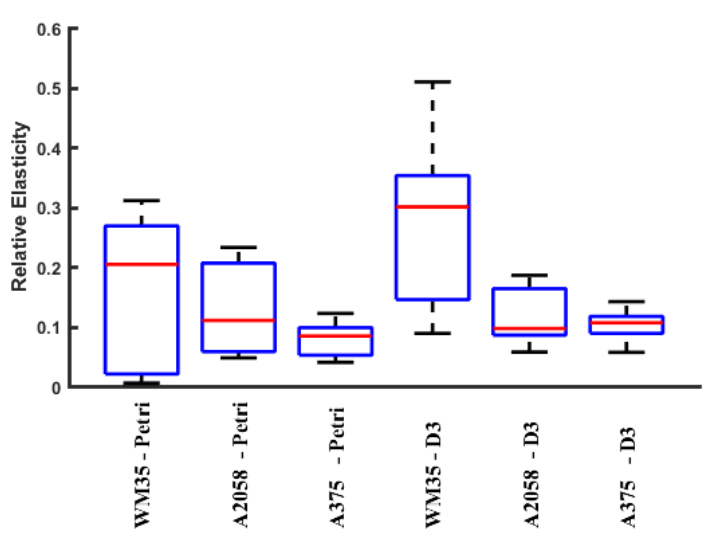

Figure 3 Comparison of relative elasticity of the three studied cell types, when pushed towards a bare Petri dish surface and a confluent endothelial layer successively.

As Figure 3 depicts, a strong dependence can be observed between the relative elasticity and the melanoma cell type. WM35 cells apparently show a more elastic, while A2058 and A375 cells a more plastic behaviour upon deformation. The slightly higher values when two cells are pushed together might be attributed to the presence of the endothelium.

In order to characterize the linkage strength between the melanoma cells and the endothelium, first the maximal adhesion force was calculated. 


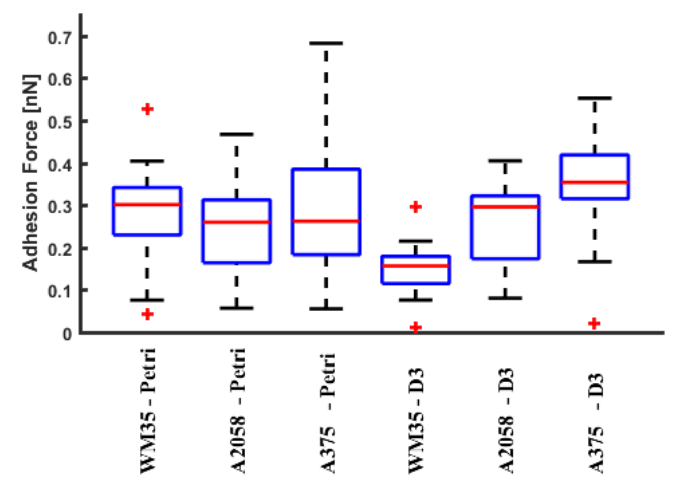

Figure 4 Maximal adhesion force between the studied cell types when pushed to a bare Petri dish surface and a confluent endothelial layer, respectively. Plus signs represent outlier values.

When the melanoma cells are pushed to a bare Petri dish, all show similar adhesion strength, while a clear dependence on cell type can be observed in case of endothelium (see Figure 4). If we compare the results in Figure 3 with those in Figure 4, one can observe that the relative elasticity is inversely correlated with maximal adhesion force.

As the de-adhesion process is not continuous, individual rupture like events can be distinguished on the recorded force plots (see Figure 2 for explanation and example). Figure 5 shows the number of these events for the studied melanoma cells. In both recorded sets the dependence is rather similar, more aggressive cells establish more active connections while brought into contact with same load and dwell time.

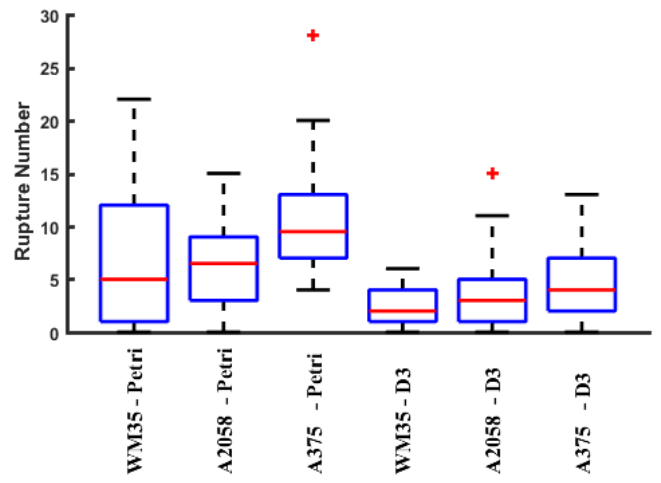

Figure 5 Comparison of occurred number of rupture events per force curve for the three studied cell types, when pushed to a bare Petri surface and a confluent endothelial layer. Plus signs represent outlier values.

The exact reason why higher number of ruptures can be identified when the melanoma cells are pushed to a bare Petri dish compared when brought in contact with the endothelium is still under debate. Relative elasticity can be an important factor in this process, but the exact reasons are unclear. However, a clear and strong correlation can be observed which is similar for both studied cases assuming that the most formed connections occur in case of A375 cells, while the least are for the WM35 cells.

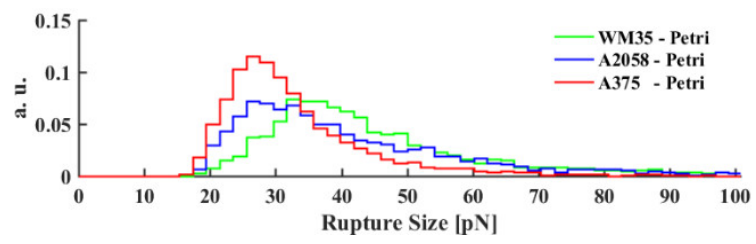

Figure 6 Rupture size distribution of the studied melanoma cells when pushed to a bare Petri dish surface.

Besides the number of apparent bonds their size and occurring place (length) carries important information as well. The apparent rupture size can be associated mostly with the molecular background of the studied cells. Similar distribution pattern can be observed when the melanoma cells are pushed to Petri dish (Figure 6) and confluent endothelium (Figure 7). The most frequent values are around $30 \mathrm{pN}$, which is independent of the substrate. The relative abundance varies with the measured cell 
type, from WM35 with lower values to A375 with higher frequency values showing a slight shift towards lower values.

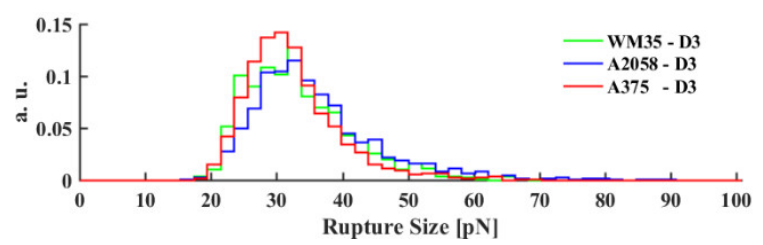

Figure 7 Rupture size distribution of the studied melanoma cells when pushed to a confluent layer of endothelial cells.

Even more interesting feature is at what distance occur these rupture events from the contact point. The distribution of these distances in case of melanoma-Petri and melanomaendothelium are plotted in Figure 8 and Figure 9. As the cells undergo a slight shape change during their contact (mostly the melanoma, as the endothelial cell is adhered and flattened), the highest peaks can be observed below one micrometre.

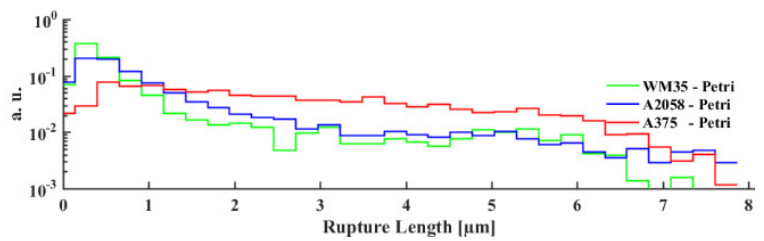

Figure 8 Probability distribution of the rupture length for the de-adhesion events occurred during melanoma cell - Petri dish contact.
This is the zone where simultaneous events occur and their number is dependent on the contact area.

After the initial quick shrinkage of this area, rupture length distribution can be mainly associated with membrane dynamics, and spontaneous unlocking of cell adhesion molecules and other molecular cross talks.

The higher frequencies of values below one micrometre can be associated with the apparent relative elasticity of the melanoma cells. As they appear more elastic, they regain their original size quicker, resulting most of de-adhesion events at close distances to contact point.

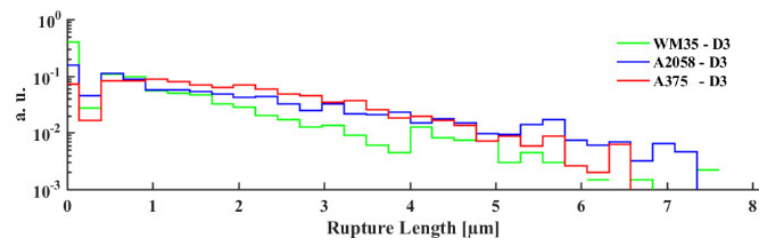

Figure 9 Probability distribution of the rupture length for the de-adhesion events occurred in case of melanoma cell - endothelial interaction.

De-adhesion events occurring at high distances (more than 2 micrometre from contact point) can be predominantly associated with membrane tether formations. In both cases, melanoma cell - Petri and melanoma cell endothelial interactions.

\section{Discussion \& Summary}

Arrest of melanoma cells on the inner surface of the brain blood vessels is a crucial but not a sufficient step in the process of brain metastasis formation. Nevertheless, those blood-traveling melanoma cells which show higher adhesiveness to the brain endothelium might have higher chance to successfully colonize the brain. The mechanism of melanoma cell arrest and establishment of firm contact to brain endothelial cells is still only partially described and understood. Here we show a comparison of the dynamics of the first contact of three types of melanoma cells (WM35, A2058 and A375) with brain endothelial cells. The WM35 is a cutaneous, non-metastatic cell line, while A2058 and A375 are highly metastatic cell lines (25). The difference in the metastatic potential between the A2058 cells and A375 cells has not been clearly established so far. Both cell lines are VCAM-1 negative (26), however, Rolland et al. found that transmigration of A2058 cells across bovine brain capillary endothelial cells, was twice as effective compared to A375 (27). Both A2058 and A375 cells show similar adhesive properties to brain endothelial cells with similar junction damaging potential in static models $(28,29)$. Pogoda et al. denotes A375 cells as highly invasive (30) and compares its elasticity to WM35 emphasizing that the former has lower elastic modulus (which correlates well with our results). The above mentioned studies deal with the 
total transmigration process of the melanoma cells across the endothelium, which includes but is not restricted to initial affinity dynamics of firm adhesion establishment. Furthermore, these studies were conducted on static conditions.

Intercellular adhesion dynamics depends on multiple factors, amongst which we can find apparent whole cell elasticity, visco-elastic properties, surface charge density, surface linked adhesion molecule distribution and glycocalyx thickness as well. The parameters investigated by us include plasticity, maximal adhesion force, size and location of de-adhesive rupture events. Referring to the first short term contact to brain endothelial cells our data suggests the following order from lower to higher metastatic potential - WM35, A2058 and A375 - based on apparent affinity to brain endothelial cells.

Unfortunately, no proper model exists to obtain elastic or plastic properties when two cells are pushed against each other. Therefore, in order to compare the elastic properties of the studied cell types in situ. similarly to the plasticity index (23) we have used the relative elasticity, or elastic index, as a dimensionless comparing factor, which consists of the ratio between the remanent and total work needed to obtain the pre-set load (for details see section of Materials and Methods). In our case the perfect elasticity would be 1 while total plasticity 0 . As presented in Figure 3 the obtained relative elasticity values are similar both in the case when melanoma cells were pushed against the Petri dish surface and against the endothelial layer. This indicates that the calculated relative elasticity is predominantly the property of the melanoma cells and the endothelial cells have low contribution. The highest relative elasticity values were shown by WM35 cells, followed by A2058 and A375 cells respectively. These results are in line with the findings of Lekka et al., which demonstrate a higher relative Young's modulus (decreased elasticity) for WM35 cells compared to A375 cells (31).

Cell elasticity is mainly determined by cytoskeletal structures and low elasticity may reflect disorganization of the cytoskeletal characteristic to aggressive cancer cells (32). Besides biomechanical properties of a cell, physical aspects of cell-cell interaction may influence cellular behavior, too. The force necessary to move apart two cells (adhesion force) reflects well the strength of the connection. Maximal adhesion force is a well-established parameter to characterize adhesion properties of biological samples, ranging from individual molecules to living cells (10). Figure 4 shows the comparison of the calculated maximal adhesion forces between the three studied cell types contacting a bare Petri dish and the endothelial layer. In case of the Petri dish, no differences can be found between the investigated cells suggesting a non-specific interaction between the plastic surface of the Petri dish and the cells. In contrast, when melanoma cells were pushed against the endothelium a clear difference can be observed. WM35 cells show the lowest adhesion force, higher values can be observed in the case of A2058 cells, whereas the A375 cells show the highest adhesion forces from brain endothelial cells. This indicated that the more aggressive melanoma cell types adhere stronger to the cerebral endothelium possibly leading to an enhanced transmigratory and metastasis forming capacity. Interestingly, -although independent of cell type - adhesion forces are higher in case of Petri dish - melanoma cell contacts compared to melanoma cell - endothelial cell contact. An explanation of this observation could be the specially treated, cell culture grade plastic surface to which cultured cells can easily adhere.

The process of de-adhesion is not continuous; it can be decomposed into a series of de-adhesion events. Intercellular adhesion is largely determined by specific cell-cell adhesion molecules and non-specific interactions of the glycocalyx. When pulling apart two adhering cells these interactions have to be released. In addition, membrane nanotube formation can also be observed during the release of the contact. Comparing the number of the observed rupture events (Figure 5), which is directly related to active contact points, the same relation can be observed as for the maximal adhesion force. This implies the presence of a surface size related active binding process, which has higher weighting in case of more inelastic cells (see Figure 3). Size distribution of the active de-adhesive events is depicted in Figure 6 and Figure 7 for melanoma - Petri surface and melanoma - endothelial contact respectively. The most abundant values are around $30 \mathrm{pN}$, presenting a slight down-shift with the cell type for both cases. According to literature, this value is associated with de-coupling of membrane bound adhesion molecules in case of membrane tether 
ruptures (33), although it is very close to the adhesion forces reported in case of E-cadherin fragments (34).

Additionally, the occurrence place of these de-adhesion events is an important characteristic of membrane dynamics, since in many cases they appear at several micrometre distances from the contact point. Based on our data, we can conclude that the shift towards higher distances of occurrence places of detected deadhesion events (Figure 8 and Figure 9) might indicate that the role of tether based adhesive properties of invading melanoma cells cannot be neglected in the metastasis formation process. Tether formation and dynamics might contribute considerably to site selection of melanoma cells ending in successful arrest on the surface of brain endothelial layer. Although it is not an easy task to quantify the weighting of tether based adhesive contribution within the full detachment force, it might grant metastatic melanoma cells one step forward to successful colonization.

\section{Conclusion}

Finally, as a conclusion we can say that we have used successfully the AFM based single cell spectroscopy for comparison and analysis of adhesion force dynamics between a confluent brain endothelial layer and three different type of melanoma cells presenting different invasive characteristics. Apparent mechanical properties such as elasticity, maximal adhesion force, number, size and distance of individual rupture events showed altered values pointing towards cell type dependent aspects. Our results underline the importance of mechanical details in case of intercellular interactions. Nevertheless, it suggests that in adequate circumstances elastic and adhesive characterizations might be used as biomarkers.

\section{Acknowledgement}

This work was supported by the National Science Fund of Hungary OTKA K116158, PD115697, PD121130, the GINOP-2.3.2-15-2016-00001 / 00020 / 00034 and GINOP-2.3.3-15-2016-00030 programs. Imola Wilhelm and Attila G. Végh were supported by the János Bolyai Fellowship of the Hungarian Academy of Sciences BO/00334/16/8 and BO/00598/14/8 respectively.

The authors declare no conflict of interest.

\section{References}

1. Paluncic, J., Z. Kovacevic, P.J. Jansson, D. Kalinowski, A.M. Merlot, M.L.-H. Huang, H.C. Lok, S. Sahni, D.J.R. Lane, and D.R. Richardson. 2016. Roads to melanoma: Key pathways and emerging players in melanoma progression and oncogenic signaling. Biochim. Biophys. Acta BBA - Mol. Cell Res. 1863: 770-784.

2. Abildgaard, C., and P. Guldberg. 2015. Molecular drivers of cellular metabolic reprogramming in melanoma. Trends Mol. Med. 21: 164-171.

3. Batus, M., S. Waheed, C. Ruby, L. Petersen, S.D. Bines, and H.L. Kaufman. 2013. Optimal management of metastatic melanoma: current strategies and future directions. Am. J. Clin. Dermatol. 14: 179-194.

4. Fidler, I.J., G. Schackert, R.D. Zhang, R. Radinsky, and T. Fujimaki. 1999. The biology of melanoma brain metastasis. Cancer Metastasis Rev. 18: 387-400.

5. Jang, S., and M.B. Atkins. 2016. Treatment of Melanoma CNS Metastases. Cancer Treat. Res. 167: 263-279. 
6. Wilhelm, I., J. Molnar, C. Fazakas, J. Hasko, and I.A. Krizbai. 2013. Role of the blood-brain barrier in the formation of brain metastases. Int. J. Mol. Sci. 14: 1383-1411.

7. Kumar, S., and V.M. Weaver. 2009. Mechanics, malignancy, and metastasis: The force journey of a tumor cell. Cancer Metastasis Rev. 20: 113-127.

8. Ahmad Khalili, A., and M.R. Ahmad. 2015. A Review of Cell Adhesion Studies for Biomedical and Biological Applications. Int. J. Mol. Sci. 16: 18149-18184.

9. Yallapu, M.M., K.S. Katti, D.R. Katti, S.R. Mishra, S. Khan, M. Jaggi, and S.C. Chauhan. 2015. The roles of cellular nanomechanics in cancer. Med. Res. Rev. 35: 198-223.

10. Helenius, J., C.-P. Heisenberg, H.E. Gaub, and D.J. Muller. 2008. Single-cell force spectroscopy. J. Cell Sci. 121: 1785-1791.

11. Panorchan, P., M.S. Thompson, K.J. Davis, Y. Tseng, K. Konstantopoulos, and D. Wirtz. 2006. Single-molecule analysis of cadherin-mediated cell-cell adhesion. J. Cell Sci. 119: 66-74.

12. Varga, B., C. Fazakas, J. Molnár, I. Wilhelm, R.A. Domokos, I.A. Krizbai, Z. Szegletes, G. Váró, and A.G. Végh. 2016. Direct mapping of melanoma cell - endothelial cell interactions. J. Mol. Recognit. JMR. .

13. Omidvar, R., M. Tafazzoli-Shadpour, M.A. Shokrgozar, and M. Rostami. 2014. Atomic force microscope-based single cell force spectroscopy of breast cancer cell lines: an approach for evaluating cellular invasion. J. Biomech. 47: 3373-3379.

14. Sundar Rajan, V., V.M. Laurent, C. Verdier, and A. Duperray. 2017. Unraveling the ReceptorLigand Interactions between Bladder Cancer Cells and the Endothelium Using AFM. Biophys. J. 112: 1246-1257.

15. Hofschröer, V., K.A. Koch, F.T. Ludwig, P. Friedl, H. Oberleithner, C. Stock, and A. Schwab. 2017. Extracellular protonation modulates cell-cell interaction mechanics and tissue invasion in human melanoma cells. Sci. Rep. 7: 42369.

16. Malek-Zietek, K.E., M. Targosz-Korecka, and M. Szymonski. 2017. The impact of hyperglycemia on adhesion between endothelial and cancer cells revealed by single-cell force spectroscopy. J. Mol. Recognit. JMR. .

17. Weksler, B.B., E.A. Subileau, N. Perrière, P. Charneau, K. Holloway, M. Leveque, H. TricoireLeignel, A. Nicotra, S. Bourdoulous, P. Turowski, D.K. Male, F. Roux, J. Greenwood, I.A. Romero, and P.O. Couraud. 2005. Blood-brain barrier-specific properties of a human adult brain endothelial cell line. FASEB J. Off. Publ. Fed. Am. Soc. Exp. Biol. 19: 1872-1874.

18. Hutter, J.L., and J. Bechhoefer. 1993. Calibration of atomic-force microscope tips. Rev. Sci. Instrum. 64: 3342-3342.

19. Sader, J.E., J.A. Sanelli, B.D. Adamson, J.P. Monty, X. Wei, S.A. Crawford, J.R. Friend, I. Marusic, P. Mulvaney, and E.J. Bieske. 2012. Spring constant calibration of atomic force microscope cantilevers of arbitrary shape. Rev. Sci. Instrum. 83: 103705.

20. Higgins, M.J., R. Proksch, J.E. Sader, M. Polcik, S. Mc Endoo, J.P. Cleveland, and S.P. Jarvis. 2006. Noninvasive determination of optical lever sensitivity in atomic force microscopy. Rev. Sci. Instrum. 77: 013701.

21. Végh, A.G., C. Fazakas, K. Nagy, I. Wilhelm, J. Molnár, I.A. Krizbai, Z. Szegletes, and G. Váró. 2012. Adhesion and stress relaxation forces between melanoma and cerebral endothelial cells. Eur. Biophys. J. EBJ. 41: 139-145.

22. Zhang, X., A. Chen, D. De Leon, H. Li, E. Noiri, V.T. Moy, and M.S. Goligorsky. 2003. Atomic force microscopy measurement of leukocyte-endothelial interaction. Am. J. Physiol. - Heart Circ. Physiol. 286: H359-H367. 
23. Briscoe, B.J., and K.S. Sebastian. 1996. The Elastoplastic Response of Poly(Methyl Methacrylate) to Indentation. Proc. R. Soc. Lond. Math. Phys. Eng. Sci. 452: 439-457.

24. Williamson, D.F., R.A. Parker, and J.S. Kendrick. 1989. The box plot: a simple visual method to interpret data. Ann. Intern. Med. 110: 916-921.

25. Cardile, V., G. Malaponte, C. Loreto, M. Libra, S. Caggia, F.M. Trovato, and G. Musumeci. 2013. Raf kinase inhibitor protein (RKIP) and phospho-RKIP expression in melanomas. Acta Histochem. 115: 795-802.

26. Jonjic, N., I. Martìn-Padura, T. Pollicino, S. Bernasconi, P. Jílek, A. Bigotti, R. Mortarini, A. Anichini, G. Parmiani, F. Colotta, E. Dejana, A. Mantovani, and P.G. Natali. 1992. Regulated Expression of Vascular Cell Adhesion Molecule-1 in Human Malignant Melanoma. Am. J. Pathol. 141: 1323-1330.

27. Rolland, Y., M. Demeule, L. Fenart, and R. Béliveau. 2009. Inhibition of melanoma brain metastasis by targeting melanotransferrin at the cell surface. Pigment Cell Melanoma Res. 22: 8698.

28. Fazakas, C., I. Wilhelm, P. Nagyoszi, A.E. Farkas, J. Hasko, J. Molnar, H. Bauer, H.C. Bauer, F. Ayaydin, N.T. Dung, L. Siklos, and I.A. Krizbai. 2011. Transmigration of melanoma cells through the blood-brain barrier: role of endothelial tight junctions and melanoma-released serine proteases. PloS One. 6: e20758.

29. Molnár, J., C. Fazakas, J. Haskó, O. Sipos, K. Nagy, Á. Nyúl-Tóth, A.E. Farkas, A.G. Végh, G. Váró, P. Galajda, I.A. Krizbai, and I. Wilhelm. 2016. Transmigration characteristics of breast cancer and melanoma cells through the brain endothelium: Role of Rac and PI3K. Cell Adhes. Migr. 10: 269-281.

30. Pogoda, K., J. Jaczewska, J. Wiltowska-Zuber, O. Klymenko, K. Zuber, M. Fornal, and M. Lekka. 2012. Depth-sensing analysis of cytoskeleton organization based on AFM data. Eur. Biophys. J. EBJ. 41: 79-87.

31. Lekka, M., K. Pogoda, J. Gostek, O. Klymenko, S. Prauzner-Bechcicki, J. Wiltowska-Zuber, J. Jaczewska, J. Lekki, and Z. Stachura. 2012. Cancer cell recognition - Mechanical phenotype. Micron. 43: 1259-1266.

32. Smolyakov, G., B. Thiebot, C.C. Campillo, S. Labdi, C. Séverac, J. Pelta, and E. Dague. 2016. Elasticity, adhesion and tether extrusion on breast cancer cells provide a signature of their invasive potential. ACS Appl. Mater. Interfaces. .

33. Benoit, M., and C. Selhuber-Unkel. 2011. Measuring cell adhesion forces: theory and principles. Methods Mol. Biol. Clifton NJ. 736: 355-377.

34. du Roure, O., A. Buguin, H. Feracci, and P. Silberzan. 2006. Homophilic interactions between cadherin fragments at the single molecule level: An AFM study. Langmuir. 22: 4680-4684. 